\title{
Desempenho de algoritmos de correção atmosférica sobre dados de reflectância aplicados ao monitoramento remoto da qualidade das águas continentais
}

\author{
William Gaida, Joceli Augusto Gross, Gustavo Rodrigues Toniolo
}

https://doi.org/10.4322/mp.978-65-991393-8-3.c4

\begin{abstract}
Resumo
O uso de imagens de sensores orbitais representa uma alternativa viável para o monitoramento de recursos hídricos. No entanto, o desempenho da aplicação de dados obtidos por meio de técnicas de sensoriamento remoto depende do conhecimento de suas limitações e possíveis erros associados aos dados, como os efeitos atmosféricos, que correspondem a uma das maiores fontes de incerteza das medições extraídas de dados de sensoriamento remoto, sendo fundamental sua correção para a obtenção de medidas radiométricas representativas das características espectrais dos alvos analisados. Este estudo tem como objetivo avaliar o desempenho de algoritmos de correção atmosférica aplicados a imagens para estimar a refletância da superfície da água. $O$ estudo foi realizado em um compartimento aquático localizado na margem esquerda do reservatório de Itaipu. Quatro campanhas de campo foram conduzidas para aquisição de medidas de refletância de superfície da água em treze estações amostrais. Uma série temporal de imagens do sistema sensor Operational Land Imager coincidentes com as datas das campanhas de campo foi adquirida, sendo estas processadas a partir da aplicação dos algoritmos de correção atmosférica LaSRC, FLAASH, iCOR e QUAC. O desempenho dos diferentes algoritmos foi avaliado utilizando como referência as medidas radiométricas obtidas in situ, sem a influência de efeitos atmosféricos. Todos os algoritmos tiveram melhor desempenho em relação à reflectância de referência nos dados da banda do vermelho do sensor OLI (banda OLI B4), o que indica que os dados desta banda se mostraram menos influenciados pelos efeitos atmosféricos.
\end{abstract}

Palavras-chave: ambientes aquáticos, efeitos atmosféricos, Landsat-8, sensoriamento remoto.

\section{Introdução}

O sensoriamento remoto é a principal fonte de informação espacial sobre a cobertura e a constituição da superfície terrestre [1]. Nas últimas décadas, o uso desta geotecnologia consolidou-se como uma alternativa eficaz aos métodos tradicionais de monitoramento de ambientes aquáticos baseados em amostragem pontual in situ [2]. Neste aspecto, as imagens da série de satélites Landsat têm sido extensamente utilizadas em estudos com as mais diversas abordagens sobre mapeamento e monitoramento de ambientes aquáticos [3]. Características como a resolução espacial nominal de 30 metros e radiométrica de 16 bits permitem a aplicação de imagens do sistema sensor multiespectral Operational Land Imager (OLI), a bordo do satélite Landsat-8, no estudo de superfícies aquáticas continentais [4]. 
Embora os dados adquiridos por meio de técnicas de sensoriamento remoto representem um enorme potencial como ferramenta no auxílio ao monitoramento de recursos hídricos continentais, sua eficiência é dependente do conhecimento de suas limitações e erros acoplados ao sinal detectado pelo sistema sensor. Neste sentido, os efeitos atmosféricos, decorrentes da interação da radiação eletromagnética proveniente do Sol com os componentes atmosféricos opticamente ativos, como partículas e moléculas gasosas, representam uma das fontes de incerteza radiométrica mais significativa ao aspecto de qualidade dos dados de sensoriamento remoto em aplicações com foco nas propriedades físicas, químicas e biológicas dos alvos $[5,6]$. Sobre superfícies aquáticas estes efeitos são mais agravados devido à baixa reflectância característica da água, o que faz com que a maior parte da radiância detectada pelos sensores corresponda a efeitos de espalhamento atmosférico [7].

A correção dos efeitos atmosféricos em imagens de sensores orbitais consiste em uma etapa fundamental para o desenvolvimento de análises quantitativas precisas a partir de medidas de reflectância de superfície [8]. A correção atmosférica consiste na remoção dos efeitos de atenuação da radiação eletromagnética, causados pelos constituintes atmosféricos opticamente ativos, como a dispersão molecular causada por aerossóis e a absorção provocada pelas moléculas de vapor de água, ozônio, oxigênio e dióxido de carbono [9].

Tendo em vista a necessidade da correção destes efeitos, diversos métodos têm sido propostos a partir da aplicação de diferentes abordagens [10]. Desta forma, os métodos de correção atmosférica podem ser divididos em três categorias principais: métodos absolutos, fundamentados em princípios físicos que utilizam códigos de transferência radiativa para a determinação da influência dos componentes atmosféricos na radiância detectada por um sistema sensor; métodos relativos, que estimam a contribuição dos efeitos atmosféricos por meio de informações obtidas a partir dos metadados e/ou reflectância de alvos da própria imagem; e métodos híbridos que possuem versões simplificadas dos códigos de transferência radiativa e realizam a aquisição das informações necessárias a partir da própria imagem [11-13].

Diversos estudos, como os realizados por Bonansea et al. [14], Bernardo et al. [15] e Martins et al. [16] têm sido conduzidos com o objetivo de avaliar o desempenho de diversos algoritmos para a correção atmosférica de imagens de sensoriamento remoto. No entanto, estes estudos não são conclusivos a respeito de qual modelo detém, de forma definitiva, o melhor desempenho, devido à incerteza associada à interação entre a composição atmosférica e a composição da água. A correção efetiva dos efeitos atmosféricos sobre superfícies aquáticas continentais mostra-se uma tarefa desafiadora, devido a fatores como a continentalidade atmosférica e as particularidades características de cada ambiente aquático [7]. Desta forma, a necessidade da realização de avaliações prévias deve ser considerada, principalmente, em estudos sobre áreas com resposta espectral complexa como reservatórios artificiais.

Neste sentido, o presente estudo tem como objetivo avaliar o desempenho de diferentes algoritmos de correção atmosférica sobre medidas de reflectância detectadas pelo sistema sensor OLI, aplicadas ao monitoramento da qualidade de águas continentais.

\section{Material e Métodos}

\section{1. Área de estudo}

Este estudo foi conduzido em um compartimento do Reservatório de Itaipu formado pela foz do Rio São Francisco Verdadeiro. Está localizado no Sul da linha do equador entre as

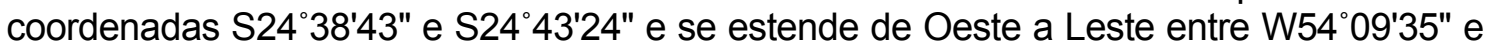


W54 19'03". É delimitado ao Norte pelo município de Pato Bragado, ao Sul pelo município Entre Rios do Oeste, a Leste pelos municípios de Marechal Cândido Rondom e Cascavel e a Oeste pelo corpo principal do lago Itaipu (Figura 1).
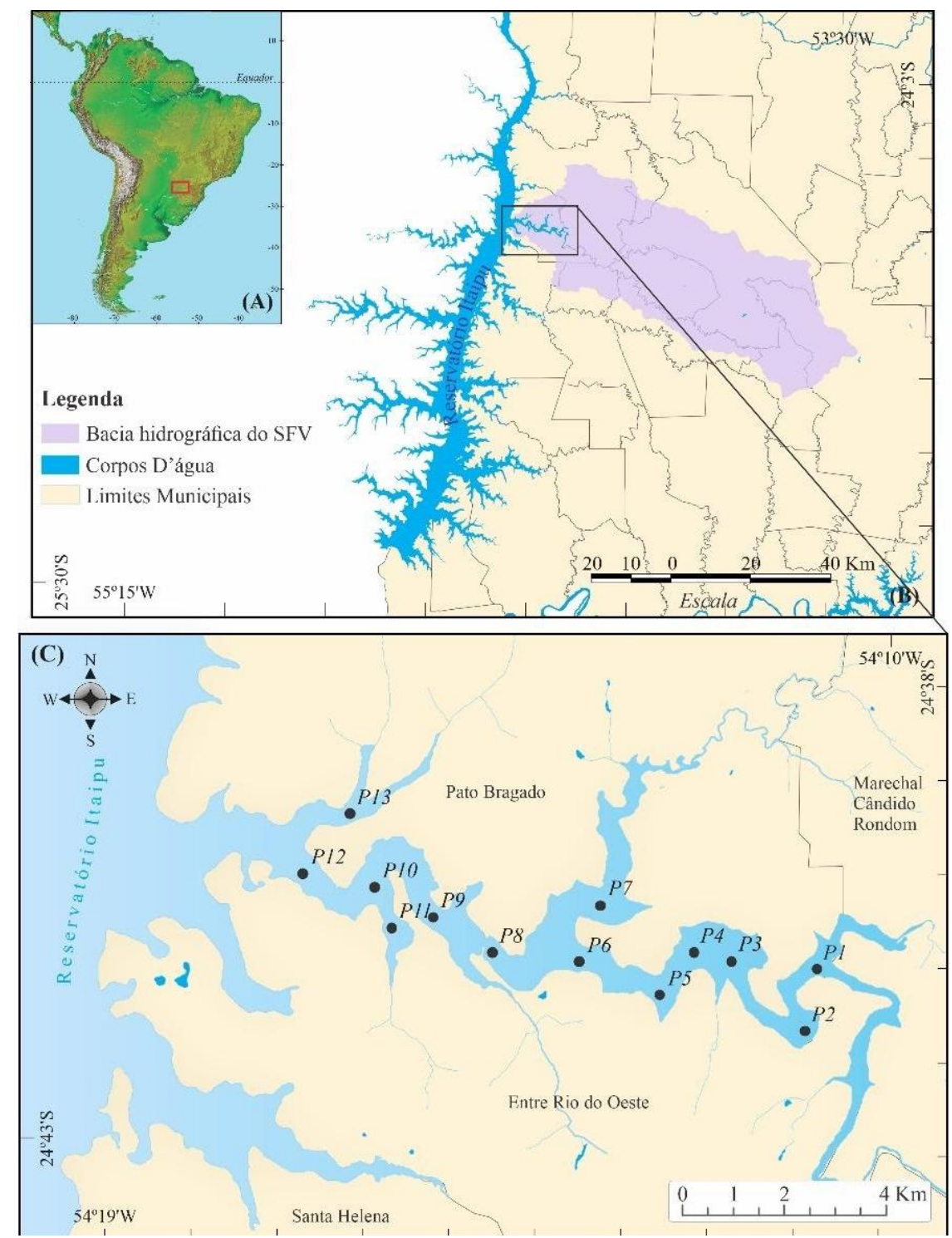

Figura 1. Mapa de localização da área de estudo, (A) na América do Sul, (B) no reservatório de Itaipu e $(C)$ localização das estações amostrais utilizadas para a aquisição dos dados de campo.

A área compreendida pela lâmina de água do compartimento do Rio São Francisco Verdadeiro é de aproximadamente $21,5 \mathrm{~km}^{2}$, sendo sua nascente localizada na área urbana do município de Cascavel. Suas águas são fortemente caracterizadas pela influência de sua bacia hidrográfica, que drena uma área de aproximadamente 2.219,11 $\mathrm{km}^{2}$, sendo a maior bacia tributária da margem esquerda do Reservatório de Itaipu.

\subsection{Aquisição e processamento de imagens de sensoriamento remoto}

De forma paralela à realização das campanhas de campo, duas séries de imagens Landsat-8/OLI foram adquiridas nos níveis de processamento L1TP (com processamentos de calibração radiométrica, correção geométrica e valores de pixel 
em números digitais) e L2 (com correção dos efeitos atmosféricos). A primeira série multitemporal de imagens foi adquirida a partir do repositório de dados Earth Explorer, mantido pela United States Geological Survey (USGS), enquanto que a segunda foi adquirida por meio do serviço de distribuição sob demanda de imagens com correção atmosférica processada pelo algoritmo Landsat Surface Reflectance Code (LaSRC) [17], disponível a partir do repositório EROS Science Processing Architecture (ESPA). Este algoritmo foi desenvolvido para otimizar o processo de distribuição de imagens do sensor OLI em nível de processamento L2, com calibração radiométrica e correção dos efeitos atmosféricos. Este algoritmo, fundamentado em princípios físicos, utiliza um código de transferência radiativa desenvolvido especialmente para as características espectrais e radiométricas do sensor OLI [17].

A série de imagens adquiridas em nível de processamento L2 não foram aplicadas rotinas de processamento, uma vez que os valores dos pixels já são disponibilizados em medidas de reflectância de superfície. Para a série em nível de processamento L1TP foram aplicados procedimentos de correção atmosférica com uso dos algoritmos Fast Line-of-sight Atmospheric Analysis of Hypercubes (FLAASH) [18], Image CORrection for atmospheric effects (iCOR) [19] e QUick Atmospheric Correction code (QUAC) [20]. O FLAASH caracteriza-se como um algoritmo de correção atmosférica fundamentado em princípios físicos, que utiliza o código de transferência radiativa MODerate-resolution atmospheric TRANsmission 4 (MODTRAN4) [21] para modelar a propagação da radiação eletromagnética pela atmosfera terrestre. Enquanto que o iCOR consiste em um algoritmo híbrido que utiliza o método Look-up table para a estimativa dos efeitos atmosféricos por meio de consulta em tabelas de parâmetros atmosféricos calculados por meio do código de transferência radiativa MODTRAN5 [22]. O QUAC consiste em um algoritmo empírico que utiliza informações obtidas a partir das medidas de reflectância de topo da atmosfera em diferentes comprimentos de onda dos alvos da imagem para a estimativa dos efeitos atmosféricos associados às medidas de reflectância de topo da atmosfera [20].

Para as aplicações dos algoritmos FLAASH e iCOR fez-se necessária a aquisição de parâmetros atmosféricos do local e data de cada uma das imagens avaliadas, obtidos a partir do repositório de dados espaciais Giovanni da NASA, disponível em: <https://giovanni.gsfc.nasa.gov/giovanni/>. Foram adquiridas medidas de profundidade óptica de aerossol, coeficiente de Ångström e espessura de coluna de vapor de água. Estas medidas foram obtidas de forma indireta por meio das medidas de reflectância espectral das bandas do sensor MODerate-resolution Imaging Spectroradiometer (MODIS), a bordo do satélite Terra, sendo disponibilizadas a partir dos produtos MOD04 MODIS/Terra level-2 Atmospheric Aerosol Product [23] e MOD05_L2 - MODIS/Terra Total Precipitable Water Vapor 5-Min L2 Swath $1 \mathrm{~km}$ and $5 \mathrm{~km}$ [24].

As medidas de profundidade óptica de aerossol e coeficiente de Ångström foram utilizadas no cálculo do coeficiente de turbidez atmosférica, obtido por meio da Equação 1 [25].

$$
\beta=\tau_{\mathrm{a}(\lambda)} \lambda^{\alpha}
$$

Onde: $\beta$ é o coeficiente de turbidez, та $(\lambda)$ é a profundidade óptica de aerossol no comprimento de onda de 550 nanômetros, $\lambda$ é o comprimento de onda em micrômetros e a é a medida do expoente de Ångström.

O valor do coeficiente de turbidez atmosférica obtido foi utilizado para o cálculo da visibilidade inicial média, utilizada na recuperação das medidas de aerossol e correção 
dos efeitos de espalhamento atmosférico. A visibilidade inicial para a área da imagem foi calculada por meio da Equação 2.

$$
\mathrm{V}=(-15) \frac{\log \left(\frac{\beta}{0,613}\right)^{2}}{2}
$$

A partir do valor médio de visibilidade inicial calculado para cada imagem e as medidas de profundidade óptica de aerossol foi calculada também a altura de escala do aerossol. Este parâmetro é utilizado pelo algoritmo FLAASH para a correção dos efeitos de adjacência espectral a partir do cálculo da reflectância espalhada de regiões adjacentes, sendo obtido por meio da Equação 3 [26].

$$
Z_{a}=\frac{\tau_{a(\lambda)}}{\frac{3,912}{V}-\beta}
$$

Os parâmetros utilizados para a correção atmosférica no algoritmo FLAASH são representados na Tabela 1. Além destes parâmetros também foram definidos 0 modelo de aerossol como rural e modelo atmosférico MODTRAN como de média latitude de verão (Mid-Latitude Summer - MLS), calibrado a partir do valor médio de espessura de coluna de vapor de água para cada uma das imagens avaliadas.

Tabela 1. Parâmetros utilizados na correção atmosférica das imagens pelo algoritmo FLAASH.

\begin{tabular}{cccccccc}
\hline Data & $\mathbf{h}(\mathbf{k m})^{\star}$ & $\begin{array}{c}\mathbf{T}_{\mathbf{a}}(\mathbf{0 , 5 5} \\
\mathbf{\mu m})\end{array}$ & $\mathbf{A}$ & $\boldsymbol{\beta}$ & $\mathbf{V}(\mathbf{k m})$ & $\boldsymbol{Z}_{\mathbf{a}}(\mathbf{k m})$ & $\begin{array}{c}\mathbf{C O}_{2} \\
(\mathbf{p p m})^{\star \star}\end{array}$ \\
\hline $08 / 06 / 2016$ & 0,312 & 0,017 & 1,557 & 0,007 & 67,737 & 0,288 & 390 \\
$11 / 08 / 2016$ & 0,312 & 0,029 & 1,500 & 0,012 & 59,217 & 0,427 & 390 \\
$11 / 06 / 2017$ & 0,312 & 0,010 & 1,500 & 0,004 & 75,188 & 0,188 & 390 \\
$13 / 07 / 2017$ & 0,312 & 0,049 & 1,500 & 0,020 & 51,350 & 0,623 & 390
\end{tabular}

*h representa a altitude média da área abrangida nas imagens.

**Medidas de dióxido de carbono atmosférico padrão do modelo MODTRAN.

Para a aplicação do algoritmo iCOR na correção atmosférica das imagens Landsat8/OLI fez-se necessária a aquisição de medidas de espessura óptica de aerossol e espessura de coluna de vapor de água para cada uma das datas das imagens, como mostrado na Tabela 2. Estes parâmetros atmosféricos foram obtidos juntamente com os parâmetros necessários para o processamento pelo algoritmo FLAASH.

Tabela 2. Parâmetros utilizados na correção atmosférica das imagens pelo algoritmo iCOR.

\begin{tabular}{ccc}
\hline Data & $\left.\mathbf{T}_{\mathbf{a}} \mathbf{( 0 , 5 5} \boldsymbol{\mu m}\right)$ & $\begin{array}{c}\text { Coluna de vapor de água } \\
\left(\mathbf{g} / \mathbf{c m}^{\mathbf{2}}\right)\end{array}$ \\
\hline $08 / 06 / 2016$ & 0,017 & 0,923 \\
$11 / 08 / 2016$ & 0,029 & 0,869 \\
$11 / 06 / 2017$ & 0,010 & 0,93 \\
$13 / 07 / 2017$ & 0,049 & 1,855 \\
\hline
\end{tabular}


A aplicação da correção atmosférica pelo algoritmo QUAC foi realizada no módulo Atmospheric Correction do software de processamento de imagens ENvironment for Visualizing Images (ENVI) versão 5.3 [27], sendo selecionados os parâmetros para o sensor OLI. Como este algoritmo procede a correção dos efeitos atmosféricos a partir da recuperação de informações sobre a reflectância de alvos na própria imagem, não foi necessária a aquisição de parâmetros atmosféricos provenientes de fontes externas.

\subsection{Aquisição de medidas de reflectância de referência}

Para a realização do estudo fez-se necessária à coleta de amostras de espectros de reflectância de referência (Figura 2). Para isso, quatro campanhas de campo foram realizadas nas datas de 08 de junho e 11 de agosto de 2016, e 11 de junho e 13 julho de 2017. As coletas foram realizadas em treze estações amostrais, distribuídas ao longo do compartimento aquático do Rio São Francisco Verdadeiro. A localização e distribuição espacial das referidas estações (Figura 1) teve como critério uma avaliação prévia das características espectrais da água ao longo do setor em estudo por meio de uma série temporal de imagens Landsat-8/OLI dos anos de 2015 e 2016.

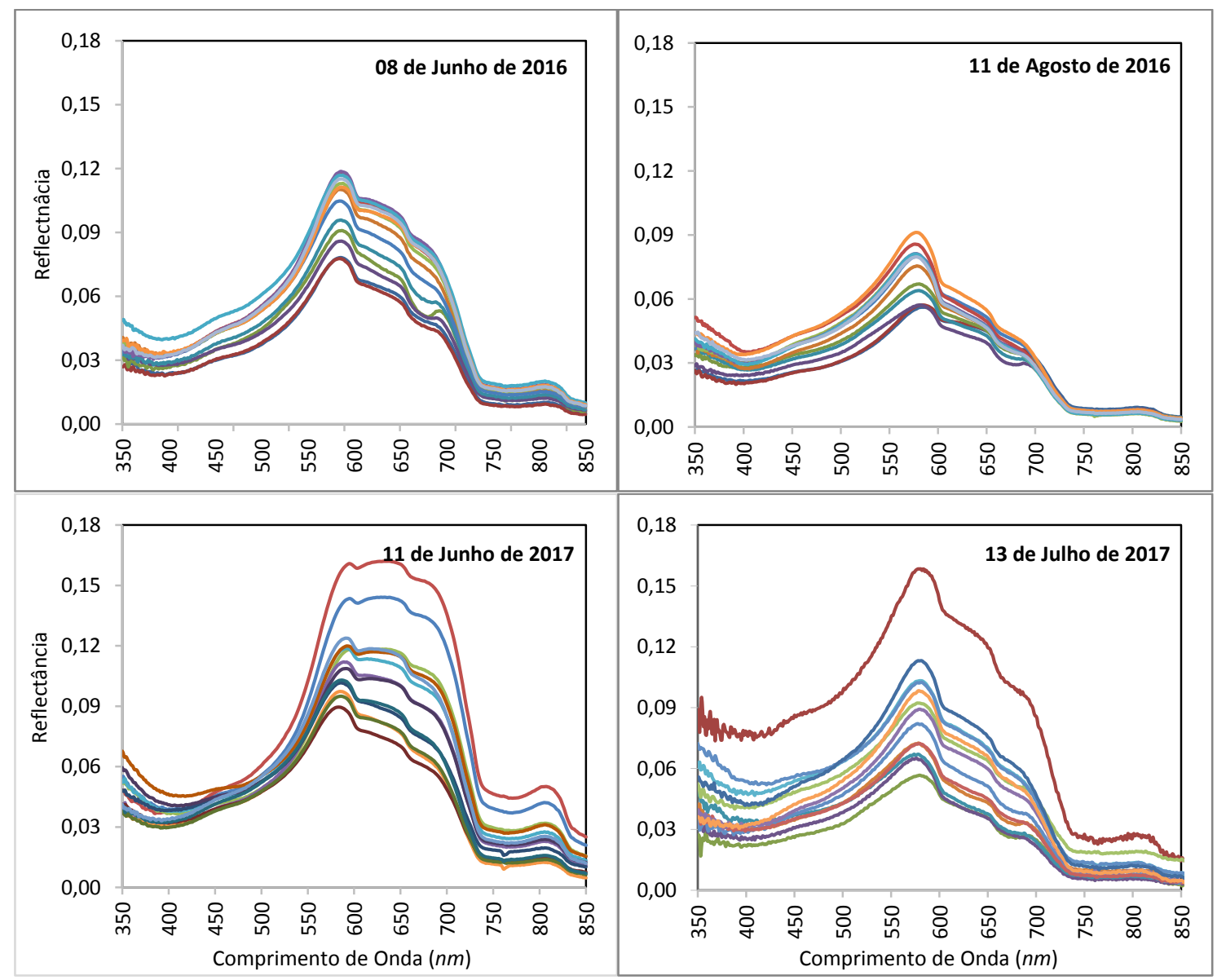

Figura 2. Espectros de reflectância obtidos durante as campanhas de campo.

A realização das campanhas de campo foi definida de modo a coincidir com as datas de imageamento da área de estudo pelo sensor OLI, de modo a evitar erros por defasagem temporal entre os dados, considerando os critérios de ausência de nebulosidade e baixa velocidade do vento. Esta última condição é descrita por Jensen (2007) como uma forma de reduzir o efeito de reflexão especular causada pela agitação da superfície da água, que prejudica a obtenção de medidas de reflectância representativas. 
Para a coleta dos espectros de reflectância foi utilizado um espectrorradiômetro FieldSpec 3, que opera entre os comprimentos de onda de 350 a 2.500 nanômetros $(\mathrm{nm})$. O aparelho foi calibrado antes de cada aquisição por meio de uma placa de referência Spectralon, sendo posicionado a um ângulo zenital de $45^{\circ}$ e azimutal de $90^{\circ}$ em relação a posição solar, a uma distância de 30 centímetros sobre a superfície da água, de modo a minimizar o efeito de reflexão especular, conforme protocolo descrito em Steffen [28]. Não foram realizadas medidas de fluxo radiante, sendo obtidas medidas de fator de reflectância bidirecional.

As medidas radiométricas obtidas foram utilizadas para a simulação da resposta do sensor OLI, conforme descrito por Mascarenhas et al. [29] e Lopes et al. [30]. Devido à proximidade com a superfície da água no momento da aquisição, estas medidas podem ser consideradas, de forma teórica, como livres da influência de efeitos atmosféricos, sendo definidas neste trabalho como padrão de referência para os valores de reflectância de superfície, as quais foram comparadas com os dados de reflectância obtidos a partir da aplicação dos algoritmos de correção atmosférica em imagens do Sensor OLI (Landsat-8).

\subsection{Avaliação e tratamento estatístico dos dados}

Após a aplicação dos processamentos de correção dos efeitos atmosféricos foram obtidas medidas de reflectância de superfície para cada uma das treze estações amostrais nas quatro datas avaliadas. Para este procedimento foram utilizados quatro arquivos em formato vetorial contendo as localizações pontuais de cada estação amostral obtidas por meio de GPS durante a realização de cada campanha de campo. A partir do pixel correspondente a localização de cada estação amostral em cada uma das quatro datas avaliadas foram adquiridas medidas de reflectância das bandas do azul (OLI2, 0,450-0,515 nm), verde (OLI3, 0,525-0,600 nm), vermelho (OLI4, 0,630 - 0,680 nm) e infravermelho próximo (OLI5, 0,845 - 0,885 nm. Estas bandas foram escolhidas por serem afetadas pelos componentes opticamente ativos das águas do Caso 2, caracterizadas pela presença de outras substâncias ou partículas além do fitoplâncton (Ex.: Gordon et al. [31]), sendo este o caso das águas do reservatório de Itaipu. O desempenho da aplicação dos algoritmos de correção atmosférica foi avaliado por meio de análise de regressão simples, considerando ajuste linear entre as medidas de reflectância obtidas pela aplicação dos algoritmos LaSRC, FLAASH, iCOR e QUAC e as medidas de reflectância de referência obtidas a partir de simulação da resposta do sensor OLI sobre os dados de reflectância coletados em campo. Além disso, foram aplicadas a análise de variância ANOVA e o teste de Tukey. Destaca-se que os dados das diferentes datas de campanhas de campo foram analisados conjuntamente.

\section{Resultados e Discussão}

A análise de variância ANOVA (Tabela 3) mostrou que ocorre pelo menos um algoritmo de correção atmosférica que gerou dados distintos da reflectância de referência. Neste sentido, a hipótese de igualdade entre os dados de reflectância de referência e dos algoritmos foi rejeitada para $\alpha=0,05$ e com $F_{\text {Crítico }}<F_{\text {Calculado. }}$.

Tabela 3. ANOVA fator único. $\alpha=0,05$.

\begin{tabular}{cccc}
\hline Bandas OLI & F calculado & valor- $P$ & F crítico \\
\hline OLI 2 & 60,698 & 0,0000 & 2,408 \\
OLI 3 & 16,031 & 0,0000 & 2,408 \\
OLI 4 & 2,474 & 0,0450 & 2,408 \\
OLI 5 & 21,776 & 0,0000 & 2,408 \\
\hline
\end{tabular}


Considerando o Teste Tukey (Tabela 4), para os dados da banda OLI B2 (faixa do azul) temos a hipótese de igualdade aceita entre os dados de reflectância de Referência/QUAC e iCOR/LaSRC. Para a banda OLI B3 (faixa do verde) a igualdade entre os dados ocorreu para FLAASH/QUAC, LaSRC/QUAC, LaSRC/FLAASH e iCOR/LaSRC. Na banda OLI B4 (faixa do vermelho) a hipótese de igualdade entre os dados pode ser aceita em todos os casos, apesar de a ANOVA ter identificado diferença em pelo menos um dos grupos de dados avaliados. Para os dados da banda OLI B5 (faixa do infravermelho próximo), a hipótese de igualdade entre os dados ocorreu entre FLAASH/Referência, LaSRC/Referência, iCOR/Referência, LaSRC/FLAASH, iCOR/FLAASH e iCOR/LaSRC.

Para os demais casos, além daqueles especificados no parágrafo anterior, o Teste Tukey identificou diferenças entre os dados. Neste sentido, deve ser destacado que nenhum dos algoritmos de correção atmosférica corrigiu a reflectância, permitindo a aceitação da hipótese de igualdade em relação aos dados de reflectância de referência em todas as bandas OLI. Porém, considerando apenas os dados dos algoritmos entre si, a hipótese de igualdade pode ser aceita entre iCOR/LaSRC em todas as bandas do sensor OLI, indicando que a correção atmosférica destes dois algoritmos resultou em medidas de reflectância semelhantes, porém distintas da reflectância de referência em determinados casos. Deve ser lembrado que o algoritmo LaSRC foi desenvolvido como padrão para a correção atmosférica das imagens do sensor OLI, então de acordo com os resultados do presente trabalho (especificamente o Teste Tukey) a escolha do algoritmo iCOR, para correção atmosférica, não resultará em diferenças significativas daqueles dados já constados nas imagens originais, o que merece maiores avaliações.

Tabela 4. Resultados obtidos a partir da aplicação do Teste Tukey. As células em cor cinza indicam diferenças entre os dados.

\begin{tabular}{c|c|c|c|c|c}
\hline \multirow{2}{*}{ Bandas OLI } & Reflectância & \multirow{2}{*}{ QUAC } & FLAASH & LaSRC & iCOR \\
& Reflectância & & & & \\
\hline \multirow{3}{*}{ OLI B2 } & Referência & 0,9098 & 0,000 & 0,000 & 0,000 \\
& QUAC & & 0,000 & 0,000 & 0,000 \\
& FLAASH & & & 0,000 & 0,000 \\
& LaSRC & & & & 0,9984 \\
\hline \multirow{4}{*}{ OLI B3 } & Referência & 0,0000 & 0,0000 & 0,0000 & 0,0017 \\
& QUAC & & 0,9997 & 0,1735 & 0,0093 \\
& FLAASH & & & 0,2514 & 0,0170 \\
& LaSRC & & & & 0,8264 \\
\hline \multirow{3}{*}{ OLI B4 } & Referência & 1,0000 & 0,0587 & 0,4978 & 0,7394 \\
& QUAC & & 0,0500 & 0,4599 & 0,7037 \\
& FLAASH & & & 0,8224 & 0,5965 \\
& LaSRC & & & & 0,9956 \\
\hline \multirow{3}{*}{ OLI B5 } & Referência & 0,0000 & 0,9994 & 0,8752 & 0,8571 \\
& QUAC & & 0,0000 & 0,0000 & 0,0000 \\
& FLAASH & & & 0,9510 & 0,9404 \\
& LaSRC & & & & 1 \\
\hline
\end{tabular}

As associações entre os dados de reflectância de referência e dos algoritmos de correção atmosférica mostraram que estes, subestimaram os dados na maioria dos casos, principalmente para os dados das bandas OLI B2 (faixa do azul) e OLI B3 (faixa do verde), conforme Figura 3. Para estas bandas, os coeficientes de determinação $\left(R^{2}\right)$ alcançaram um máximo de $R^{2}=0,545$, sendo este referente aos dados do algoritmo iCOR em associação aos dados de reflectância de referência. 
Por outro lado, para os dados das bandas OLI B4 (faixa do vermelho) e OLI B5 (faixa do infravermelho próximo), os coeficientes de determinação foram mais expressivos, principalmente nos dados da banda OLI B4, com o menor $R^{2}=0,697$, verificado para os dados de algoritmo QUAC em associação com os dados de reflectância de referência e o maior $\mathrm{R}^{2}=0,861$ para o algoritmo iCOR em associação a reflectância de referência. Para os dados da banda OLI B5 os algoritmos tiveram desempenho inferior àqueles verificados para os dados da banda OLI B4, principalmente considerando os dados de reflectância expressivos calculados pelo algoritmo de correção atmosférica QUAC como pode ser verificado na Figura 3.

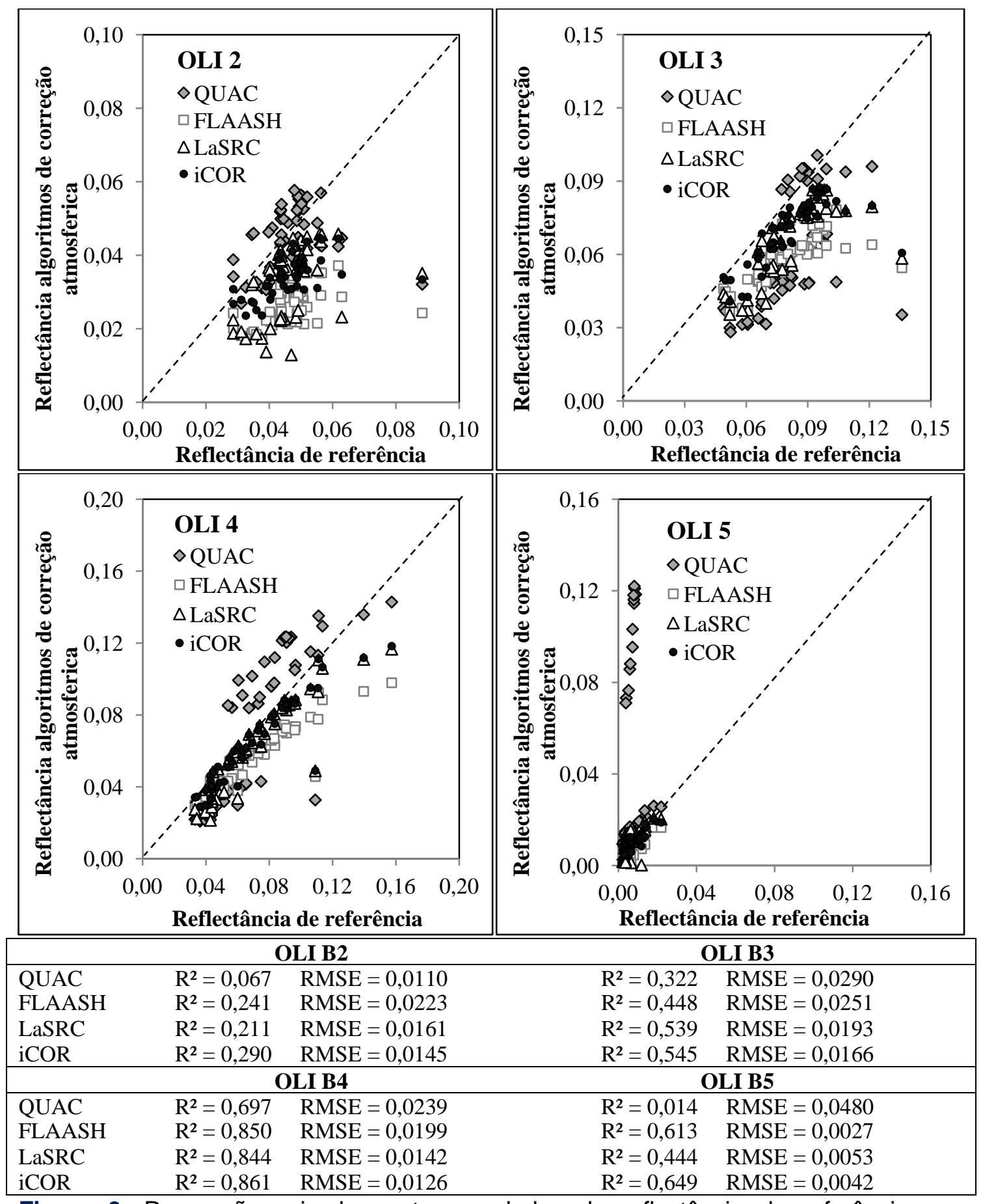

Figura 3. Regressões simples entre os dados de reflectância de referência e os calculados pelos algorítmos de correção atmosférica, além da Raiz do Erro Médio Quadrático (RMSE). 
De maneira geral todos os algoritmos tiveram melhor desempenho em relação aos dados de reflectância de referência da banda OLI B4, ou seja, na faixa do vermelho do espectro eletromagnético. Além disso, todos os algoritmos retornaram reflectâncias semelhantes entre si nos dados desta mesma banda do sensor OLI (Tabela 2). Porém, como mencionado anteriormente nenhum dos algoritmos avaliados corrigiu ou estimou valores de reflectância iguais ou semelhantes aos de referência em todas as bandas OLI (Tabela 2).

Segundo Martins et al. [16] a intensidade dos efeitos atmosféricos varia com as bandas espectrais, geometrias de aquisição e iluminação, tipos e concentração dos constituintes atmosféricos. Além disso, os autores ressaltaram que os resultados da correção atmosférica são sensíveis às interações superfície atmosfera caso a caso o que pode favorecer a erros, considerando uma mesma abordagem e que não existe consenso sobre qual o algoritmo a ser utilizado. Neste contexto, especificado por Martins et al. [16] podemos presumir que a intensidade dos efeitos atmosféricos foi menor para a banda OLI B4, na qual obteve-se no presente trabalho os melhores resultados dos algoritmos de correção atmosférica em relação aos dados de referência.

\section{Considerações finais}

Todos os algoritmos tiveram melhor desempenho em relação à reflectância de referência nos dados da banda do vermelho do sensor OLI (banda OLI B4). Nesta mesma banda, também foi verificado que todos os algoritmos de correção atmosférica calcularam reflectâncias semelhantes entre si. Esses resultados sugerem que os dados da banda OLI B4 podem ser menos afetados pelos efeitos atmosféricos, o que requer maiores avaliações. Neste sentido, este trabalho terá continuidade, visando novas avaliações com outros algoritmos de correção atmosférica, paralelamente aos avaliados no presente trabalho.

Deve ser mencionado que os dados dos algoritmos LaSRC e iCOR foram semelhantes em todas as bandas do sensor OLI, o que sugere que o algoritmo iCOR, ao ser utilizado para correção atmosférica sobre imagens do sensor OLI, as quais tem o algoritmo LaSRC como padrão para correção atmosférica, não resultará em diferenças significativas daqueles dados já constados nas imagens. Esse aspecto indica outra avaliação a ser realizada para verificar se os resultados aqui obtidos são constantes ou semelhantes em outras condições atmosféricas.

\section{Agradecimentos}

Os autores agradecem a equipe da Itaipu Binacional pelo desenvolvimento do Projeto "Utilização de sensores remotos para avaliar o índice de estado trófico da água, em uma área piloto na margem esquerda do reservatório de Itaipu" e pela aquisição e disponibilidade dos dados coletados em campo avaliados neste estudo.

\section{Referências}

[1] Schmidt K. S., Skidmore A. K. Spectral discrimination of vegetation types in a coastal wetland. Remote Sensing of Environment 2003; 85:92-108. https://doi.org/10.1016/S00344257(02)00196-7.

[2] Matthews M. W. A current review of empirical procedures of remote sensing in inland and near-coastal transitional waters. International Journal of Remote Sensing 2011; 32:6855-6899. https://doi.org/10.1080/01431161.2010.512947. 
[3] Guo M., Li, J., Sheng C., Xu J., Wu L. A review of wetland remote sensing. Sensors 2017; 17. https://doi.org/10.3390/s17040777.

[4] Irons J. R., Dwyer J. L., Barsi J. A. The next Landsat satellite: the Landsat data continuity mission. Remote Sensing of Environment 122 (2012): 11-21. https://doi.org/10.1016/j.rse.2011.08.026.

[5] De Keukelaere L., Sterckx S., Adriaensen S., Knaeps E., Reusen I., Giardino C., Bresciani M., Hunter P., Neil C., Van Der Zande D., Vaiciute D. Atmospheric correction of Landsat-8/OLI and Sentinel-2/MSI data using iCOR algorithm: validation for coastal and inland waters. European Journal of Remote Sensing 2018; 51:525-542. https://doi.org/10.1080/22797254.2018.1457937.

[6] Sola I., García-Martín A., Sandonís-Pozo L., Álvarez-Mozos J., Pérez-Cabello F., González-Audícana M., Llovería R. M. Assessment of atmospheric correction methods for Sentinel-2 images in Mediterranean landscapes. International Journal of Applied Earth Observation and Geoinformation 2018; 73:63-76. https://doi.org/10.1016/j.jag.2018.05.020.

[7] Palmer S. C. J., Kutser T., Hunter P. D. Remote sensing of inland waters: challenges, process and future directions. Remote Sensing of Environment 2015; 157:1-8. https://doi.org/10.1016/j.rse.2014.09.021.

[8] Vermote E. F., Kotchenova S. Atmospheric correction for the monitoring of land surfaces. Journal of Geophysical Research 2008; 113. https://doi.org/10.1029/2007JD009662.

[9] Gao B. C., Montes M. J., Davis C. O., Goetz A. F. H. Atmospheric correction algorithms for hyperspectral remote sensing data of land and ocean. International Journal of Remote Sensing 2009; 113:17-24. https://doi.org/10.1016/j.rse.2007.12.015.

[10] Doxani G., Vermote E., Roger J. C., Gascon F., Adriaensen S., Frantz D., Hagolle O., Hollstein A., Kirches G., Li F., Louis J., Mangin A., Pahlevan N., Pflug B., Vanhellemont Q. Atmospheric correction inter-comparison exercise. Remote Sensing 2018; 10:352. https://doi.org/10.3390/rs10020352.

[11] Lantzanakis G., Mitraka Z.; Chrysoulakis N. Comparison of physically and image based atmospheric correction methods for Sentinel-2 satellite imagery. In: Karacostas $\mathrm{T}$. S., Bais A. F., Nastos P. T. (eds). Perspectives on atmospheric sciences. Springer International Publishing: 2017, p. 255-261. Basel, Switzerland. https://doi.org/10.1007/9783-319-35095-0.

[12] Moses W. J., Sterckx, S., Montes M. J., De Keukelaere L., knaeps E. Atmospheric correction for inland waters. In: Mishra D. R., Ogashawara I., Gitelson A. A. Bio-optical modeling and remote sensing of inland waters. Elsevier: 2017, p. 69-100. Amsterdam, Netherlands.

[13] Gaida W., Breunig F. M., Galvão L. S., Ponzoni F. J. Correção atmosférica em sensoriamento remoto: uma revisão. Revista Brasileira de Geografia Física 2020; 13:229248. https://doi.org/10.26848/rbgf.v13.1.p229-248.

[14] Bonansea M., Ledesma C., Rodríguez C., Pinotti L., Antunes M. H. Effects of atmospheric correction of Landsat imagery on lake water clarity assessment. Advances in Space Research 2015; 56:2345-2355. https://doi.org/10.1016/j.asr.2015.09.018. 
[15] Bernardo N., Watanabe F., Rodrigues T., Alcântara E. Atmospheric correction issues for retrieving total suspended matter concentrations in inland waters using OLI/Landsat-8 image. Advances in Space Research 2017; 59:2335-2348. https://doi.org/10.1016/j.asr.2017.02.017.

[16] Martins V. S., Barbosa C. C. F., Carvalho L. A. S., Jorge D. S. F., Lobo F. L., Novo E. M. L. M. Assessment of atmospheric correction methods for Sentinel-2 MSI images applied to amazon floodplain lakes. Remote Sensing 2017; 9. https://doi.org/10.3390/rs9040322.

[17] Vermote E., Justice C., Claverie M., Franch, B. Preliminary analysis of the performance of the Landsat-8/OLI land surface reflectance product. Remote Sensing of Environment 2016; 185:46-56. https://doi.org/10.1016/j.rse.2016.04.008.

[18] Cooley T., Anderson G. P., Felde G. W., Hoke M. L., Ratkowski A. J., Chetwynd J. H., Gardner J. A., Adler-Golden S. M., Matthew M. W., Berk A., Bernstein L. S., Acharya P. K., Miller D., Lewis P. FLAASH a MODTRAN4-based atmospheric correction algorithm its application and validation. In: Proceedings of the IEEE Geoscience and Remote Sensing Symposium; 24-28 June 2002; Toronto. New York: IEEE Publications; 2002. p. 1414-1418.

[19] Vlaams Instituut voor Technologisch Onderzoek (VITO). iCOR plugin for SNAP toolbox: software user manual. Mol: VITO Remote Sensing Unit, 2017. 20 p.

[20] Bernstein L. S., Jin X., Gregor B., Adler-Golden S. Quick Atmospheric Correction Code: algorithm description and recent upgrades. Optical Engineering 2012; 51:111719. https://doi.org/10.1117/1.OE.51.11.111719.

[21] Berk A., Adler-Golden S. M., Ratkowski A. J., Felde G. W., Anderson G. P., Hoke M. L., Cooley T., Chetwynd J. H., Gardner J. A., Matthew M. W., Bernstein L. S., Acharya P. K., Miller D., Lewis P. Exploiting MODTRAN radiation transport for atmospheric correction: the FLAASH algorithm. In: Proceedings of the Fifth International Conference on Information Fusion; 8-11 July 2002; Annapolis. Sunnyvale: International Society of Information Fusion, 2002. p. 798-803.

[22] Berk A., Anderson G. P., Acharya P. K., Shettle E. P. MODTRAN 5.2.0.0 user's manual. Burlington: Spectral Sciences Inc.; 2008. 98 p.

[23] Levy R. C., Mattoo S., Munchak L. A., Remer L. A., Sayer A. M., Hsu N. C. The collection 6 MODIS aerossol products over land and ocean. Atmospheric Measurement Techniques Discussions 2013; 6:159-259. https://doi.org/10.5194/amt-6-2989-2013.

[24] Gao B. MODIS Atmosphere L2 Water Vapor Product. NASA MODIS Adaptative Processing System, Goddard Space Flight Center, 2015. http://dx.doi.org/10.5067/ MODIS/MOD05_L2.006.

[25] Ångström A. On the atmospheric transmission of sun radiation and in dust in the air. Geografiska Annaler 1929; 11:156-166. https://doi.org/10.2307/519399.

[26] Wong M. S., Nichol J. E., Lee K. H. Modeling of aerosol vertical profiles using GIS and remote sensing. Sensors 2009; 9:4380-4389. https://doi.org/10.3390/s90604380.

[27] Harris. ENVI version 5.3. Melbourne: Harris Geospatial Solutions, 2017. 
[28] Steffen C. A. Técnicas radiométricas com o SpectronSE-590. In: Anais do VIII Simpósio Brasileiro de Sensoriamento Remoto; 14-19 abril 1996; Salvador. São José dos Campos: INPE; 1996. p. 969-975.

[29] Mascarenhas N. D. A., Banon G. J. F., Fonseca L. M. G. Simulation of a panchromatic band by spectral linear combination of multispectral bands. In: Proceedings of IEEE International Geoscience and Remote Sensing Symposium (IGARSS); 3-6 June 1991; Espoo. New York: IEEE; 1991. p. 321-324.

[30] Lopes F. B., Novo E. M. L. M., Barbosa C. C. F., Andrade E. M., Ferreira R. D. Simulation of spectral bands of the MERIS sensor to estimate chlorophyll-a concentrations in a reservoir of the semi-arid region. Revista Agroambiente 2016; 10:96-106. http://dx.doi.org/10.18227/1982-8470ragro.v10i2.3482.

[31] Gordon H. R., Brown O. B., Evans R. H., Brown J. W., Smith R. C., Smith K. S., Baker K. S., Clark D. K. A semianalytical radiance model of ocean color. Journal of Geophysical Research 1988; 93:10909-10924. https://doi.org/10.1029/JD093iD09p10909.

\section{Autores}

William Gaida ${ }^{1, \star}$, Joceli Augusto Gross ${ }^{1}$, Gustavo Rodrigues Toniolo ${ }^{2}$

1. Departamento de Geografia, Universidade Federal de Santa Maria, Av. Roraima, 97105-900, Santa Maria, Brasil.

2. Centro Estadual de Pesquisas em Sensoriamento Remoto e Meteorologia, Universidade Federal do Rio Grande do Sul, Av. Bento Gonçalves, código postal, 91501-970, Porto Alegre, Brasil.

* Autor para correspondência: ufsm.william@gmail.com 